\title{
Blood $T_{1}$ measurements using slice-interleaved $T_{1}$ mapping (STONE) sequence
}

\author{
Steven Bellm ${ }^{*}$, Long Ngo ${ }^{1}$, Jihye Jang ${ }^{1}$, Sophie Berg ${ }^{1}$, Kraig V Kissinger ${ }^{1}$, Beth Goddu', Warren J Manning ${ }^{1,2}$, \\ Reza Nezafat ${ }^{1}$
}

From 19th Annual SCMR Scientific Sessions

Los Angeles, CA, USA. 27-30 January 2016

\section{Background}

Slice interleaved $\mathrm{T}_{1}$ (STONE) mapping sequence was recently proposed to take advantage of increased recovery time of spins to improve accuracy and precision of native myocardial $T_{1}$ values. In this sequence, a nonselective inversion pulse is followed by acquisition of the data for different slices. Therefore, blood pool may experience different recovery time at different slice location due to mixing effect and rapid blood flow movement. This may impact ECV measurements using STONE based $\mathrm{T}_{1}$ mapping sequence. While, a short $\mathrm{T} 1$ of blood after contrast allows full recovery, long native $\mathrm{T} 1$ of blood pool may cause errors in $\mathrm{T}_{1}$ measurements, which will manifest as low reproducibility and variations across different locations. Therefore, we sought to assess the native blood $T_{1}$ values measured in the right ventricle (RV) and left ventricle (LV) by studying the reproducibility of $T_{1}$ measurements at different locations and slices.

\section{Methods}

Nine healthy subjects $(38 \pm 22$ years, 4 males $)$ were recruited to participate in an IRB-approved study for imaging. Each subject was in sinus rhythm and was imaged 5 times using STONE sequence with gradient echo readout (STONE-GRE) and steady-state free pre-

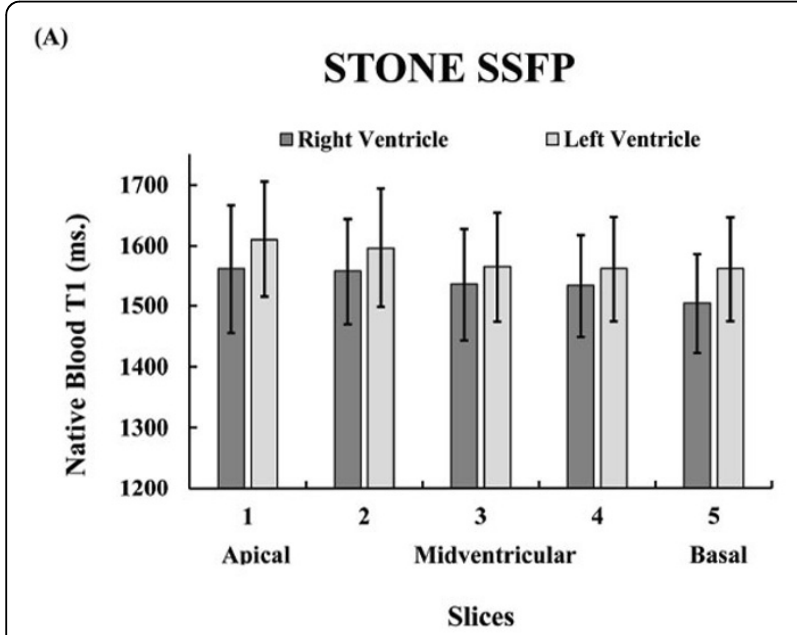

(B)

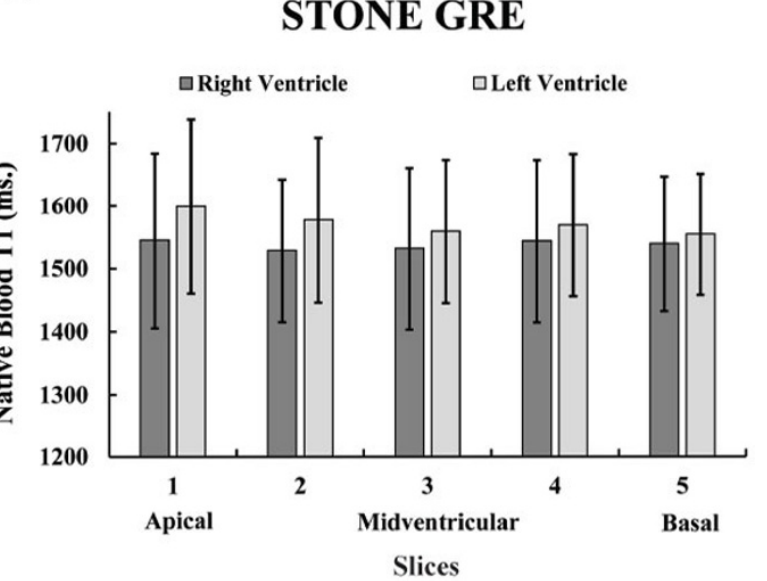

Figure 1 Mean native T1 of the blood pool per 5 slices in STONE SSFP (A) and STONE GRE (B) for RV and LV.

'Medicine, Beth Israel Deaconess Medical Center, Harvard Medical School,

Boston, MA, USA

Full list of author information is available at the end of the article

(c) 2016 Bellm et al. This is an Open Access article distributed under the terms of the Creative Commons Attribution License (http:// creativecommons.org/licenses/by/4.0), which permits unrestricted use, distribution, and reproduction in any medium, provided the original work is properly cited. The Creative Commons Public Domain Dedication waiver (http://creativecommons.org/publicdomain/ zero/1.0/) applies to the data made available in this article, unless otherwise stated. 
cession sequence (STONE-SSFP). $\mathrm{T}_{1}$ maps were reconstructed after motion correction and voxel-wise curve fitting using a 2-paramter fit model. The region of interest (ROI) for the blood pool was manually marked on five short-axis slices for RV as well as for LV to generate slice-based native $T_{1}$ values of the blood pool. Coefficient of variation (CV) analysis for each sequence was used to assess the variability of $\mathrm{T}_{1}$ measurements between each slice and between the repetitions of measurements.

\section{Results}

Figure 1 shows mean $T_{1}$ values averaged over all subjects for STONE-SSFP and STONE-GRE. $T_{1}$ means in RV were systematically smaller than in LV for both sequences $(\mathrm{p}<0.05)$. Figure 2 (A) shows a high reproducibility (GRE: $3.8 \pm 0.6 \%$, SSFP: $1.6 \pm 0.4 \%$ ) among 5 slices with significantly smaller CVs in STONE SSFP than in GRE $(\mathrm{p}<0.05)$. There was similar variability among the 5 slices for each sequence. Figure 2 (B) shows variability among the subjects with higher reproducibility for STONE SSFP (GRE: $3.7 \pm 1.5 \%$, SSFP: $1.6 \pm 0.5 \%)$.

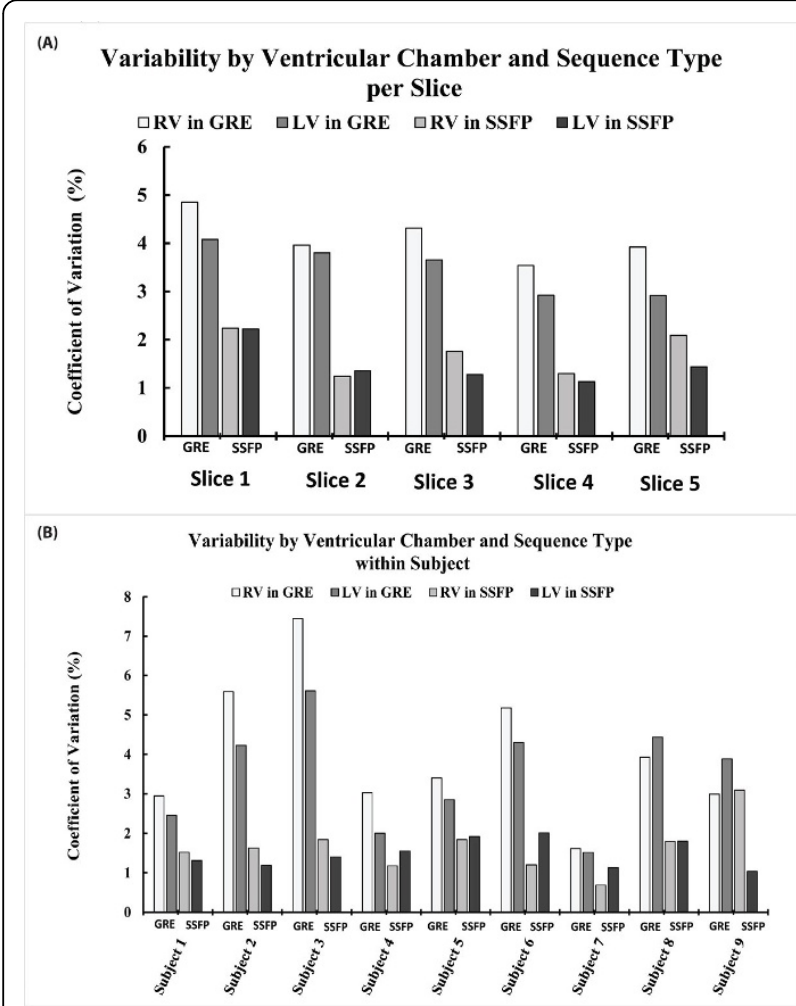

Figure 2 Variability of native T1 measurements of the blood pool by ventricular chamber and sequence type within each slice (A) and by ventricular chamber and sequence type within each subject (B)

\section{Conclusions}

Native blood $T_{1}$ measurements with STONE sequence are reproducible in both LV and RV and there is no systematic difference in $T_{1}$ measurements at difference slice locations within LV or RV. However, there are differences in $\mathrm{T}_{1}$ measurements of $\mathrm{LV}$ vs. RV for both sequences.

\section{Authors' details}

'Medicine, Beth Israel Deaconess Medical Center, Harvard Medical School, Boston, MA, USA. ${ }^{2}$ Radiology, Beth Israel Deaconess Medical Center, Harvard Medical School, Boston, MA, USA.

Published: 27 January 2016

\section{doi:10.1186/1532-429X-18-S1-P57}

Cite this article as: Bellm et al:: Blood $\mathrm{T}_{1}$ measurements using sliceinterleaved $\mathrm{T}_{1}$ mapping (STONE) sequence. Journal of Cardiovascular Magnetic Resonance 2016 18(Suppl 1):P57.
Submit your next manuscript to BioMed Central and take full advantage of:

- Convenient online submission

- Thorough peer review

- No space constraints or color figure charges

- Immediate publication on acceptance

- Inclusion in PubMed, CAS, Scopus and Google Scholar

- Research which is freely available for redistribution
Biomed Central 